Rev. Int. Contam. Ambie. 34 (4) 667-683, 2018

DOI: 10.20937/RICA.2018.34.04.09

\title{
EL ACUÍFERO YUCATECO. ANÁLISIS DEL RIESGO DE CONTAMINACIÓN CON APOYO DE UN SISTEMA DE INFORMACIÓN GEOGRÁFICA
}

\author{
Roger Amílcar GONZÁLEZ HERRERA*, Beth Sua Iztaccíhuatl ALBORNOZ EUÁN, \\ Ismael Abelardo SÁNCHEZ Y PINTO y José Humberto OSORIO RODRÍGUEZ
}

Facultad de Ingeniería, Universidad Autónoma de Yucatán, Av. Industrias no Contaminantes por Periférico Norte, tablaje catastral 12685, 97000 Mérida, Yucatán, México

*Autor para correspondencia: roger.gonzalez@correo.uady.mx

(Recibido junio 2017; aceptado febrero 2018)

Palabras clave: agua subterránea, peligro, vulnerabilidad, COST Action 620, DRSTIL, Yucatán

\section{RESUMEN}

El agua subterránea en Yucatán es la principal fuente de abastecimiento de este líquido; sin embargo, la alta permeabilidad del material geológico hace que el acuífero sea vulnerable a la contaminación. El riesgo de contaminación de acuíferos puede determinarse considerando la interacción entre las cargas contaminantes subsuperficiales y la vulnerabilidad del acuífero. El objetivo de este trabajo fue aplicar un método desarrollado para evaluar el riesgo de contaminación de aguas subterráneas que proporcione información de zonas potencialmente amenazadas. Para el análisis de riesgo se realizó un inventario de las fuentes contaminantes y se calcularon los índices de peligrosidad y de riesgo siguiendo el método del Grupo COST Action 620. Se crearon los mapas de peligrosidad y riesgo utilizando un sistema de información geográfica. Se modeló la vulnerabilidad a la contaminación del agua subterránea mediante una modificación del índice DRASTIC. El índice aplicado fue el DRSTIL (por sus siglas en inglés) donde $D$ es la profundidad del agua, $R$ la recarga, $S$ el suelo, T la topografía, I el impacto a la zona vadosa y $\mathrm{L}$ el uso del suelo. Los resultados indicaron que la vulnerabilidad a la contaminación del agua subterránea evaluada con el índice DRSTIL muestra una diferenciación más conservadora en comparación con otros estudios similares realizados en el área de estudio. La clase de vulnerabilidad moderada ocupa la mayor extensión. Las zonas de alta peligrosidad y zonas de alto riesgo se encuentran ubicadas principalmente en la zona metropolitana de Mérida.

Key words: groundwater, hazards, risk, vulnerability, COST Action 620, DRSTIL

\begin{abstract}
Groundwater in Yucatán is the main source of water supply; however, the high permeability of the geological material makes the aquifer vulnerable to contamination. The risk of aquifer contamination can be determined by considering the interaction between subsurface contaminant loads and the aquifer vulnerability. The objective of this work was to apply a methodology developed to evaluate the risk of groundwater contamination that provides information of potentially threatened areas. For the risk analysis, an inventory of contaminant sources was carried out and the hazard and risk indexes
\end{abstract}


were calculated following the methodology of the COST Action 620 Group. Hazard and risk maps were created using a geographic information system. Vulnerability to groundwater contamination was modeled by a modification of the DRASTIC index. The index applied was DRSTIL where D is water depth, $\mathrm{R}$ is recharge, $\mathrm{S}$ is soil, $\mathrm{T}$ is topography, I is impact to the vadose zone and $\mathrm{L}$ is land use. The results indicated that the vulnerability to groundwater contamination, evaluated with the DRSTIL index, shows a more conservative differentiation when compared to similar studies carried out in the study area. The moderate vulnerability class occupies the greatest extent. Areas of high danger and areas of high risk are located mainly in the metropolitan area of Mérida.

\section{INTRODUCCIÓN}

El agua subterránea es un recurso vital para los seres humanos y constituye el principal suministro de este líquido para más de 1500 millones de personas alrededor del mundo (Hudak 2005). Sin embargo, los acuíferos están experimentando una amenaza creciente de contaminación causada por la urbanización, el desarrollo industrial, las actividades agrícolas y la minería. Una vez que el acuífero se contamina, la implementación de las técnicas para la detección, el monitoreo y la remediación de la contaminación es un proceso complejo, costoso y parcialmente efectivo, por lo cual se ha dado gran énfasis a la prevención de la contaminación, la cual varía espacialmente; por ello es necesario enfocar los esfuerzos, las tecnologías y las estrategias a las áreas más amenazadas (Twarakavi y Kaluarachchi 2006).

Una herramienta útil para seleccionar los sitios a los cuales se debería dar la más alta prioridad respecto a las restricciones de actividades futuras es la evaluación del riesgo (Troldborg 2010). El enfoque de evaluación del riesgo no solamente reconoce las circunstancias ambientales, sino también los factores económicos y sociales que varían significativamente para cada acuífero; por lo tanto, establece una lógica coherente y transparente para la gestión de las aguas subterráneas mientras que permite diferentes políticas y prioridades dependiendo de las circunstancias de cada sistema (NSW 1998). Existen muchas definiciones del riesgo dependiendo de las aplicaciones específicas o de los contextos situacionales. Generalmente, se define el riesgo como la probabilidad de que un evento adverso ocurra durante un periodo de tiempo determinado o como el resultado de un cambio en particular (Troldborg 2010).

La evaluación del riesgo se refiere a la determinación de valores cualitativos y cuantitativos relacionados con un evento adverso reconocido. Aunque las prácticas y la legislación varían entre países, usualmente se adopta un procedimiento gradual en la evaluación del riesgo. La finalidad de un enfoque gradual es permitir el análisis progresivo hasta llegar a la complejidad mientras mayor sea el número de datos colectados $\mathrm{y}$, por lo tanto, reducir incertidumbres, así como la necesidad de supuestos conservadores (Troldborg 2010). La evaluación del riesgo en el enfoque gradual se realiza de acuerdo con el nivel de conocimiento y las condiciones específicas del área de estudio.

En la protección de acuíferos cársticos, la evaluación del riesgo requiere: $a$ ) identificación de peligros potenciales o mapeo de peligros existentes; $b$ ) análisis del impacto potencial de los peligros sobre el agua subterránea que requiere la concentración y cantidad de contaminantes, lo cual puede determinarse por medio de un sistema de ponderaciones y puntuaciones para cada peligro; c) información sobre las características hidrogeológicas del material geológico debajo de los peligros que influyen en el movimiento y la atenuación de los contaminantes, lo cual demuestra la vulnerabilidad del acuífero y se representa con mapas de vulnerabilidad, y d) información sobre la valoración del agua subterránea, lo que en el caso del acuífero cárstico es implícito, ya que se considera de alto valor (Zwahlen 2003).

En lo que se refiere a la contaminación del agua subterránea, el peligro se define como una fuente potencial de contaminación resultante de las actividades humanas que se desarrollan sobre la superficie del suelo. Una evaluación del peligro considera el grado potencial de contaminación de cada tipo de peligro, el cual está determinado por la toxicidad y la cantidad de sustancias nocivas que resultan del evento contaminante (Zwahlen 2003). El diseño de un inventario de fuentes potencialmente contaminantes comprende la identificación, localización espacial y caracterización sistemática de todas las fuentes, junto con la obtención de información sobre su evolución histórica donde sea apropiado y posible (Foster et al. 2002). El principal objetivo de un inventario de peligros es cubrir toda la variedad de amenazas que 
se consideran relevantes para el agua subterránea y permitir, a través de una clasificación razonable, el mapeo y la evaluación de los peligros de una manera fácil y práctica (Zwahlen 2003).

La vulnerabilidad ante la contaminación del agua subterránea se define como la tendencia o probabilidad de que los contaminantes alcancen una posición específica en el sistema hidrogeológico después de su introducción en algún lugar por encima del acuífero superior (NRC 1993). Los métodos existentes para evaluar la vulnerabilidad pueden clasificarse en tres categorías: a) métodos basados en índices, $b)$ métodos que aplican modelos determinísticos basados en procesos físicos y c) modelos estadísticos (Nobre et al. 2007). Los métodos basados en índices son atractivos porque son simples, requieren pocos datos y se implementan con facilidad en sistemas de información geográfica (SIG). Uno de los métodos de vulnerabilidad basado en índices que se utilizan frecuentemente es el DRASTIC, formulado como una suma ponderada de factores hidrológicos relacionados con el movimiento de los contaminantes de la superficie del suelo hacia los acuíferos (Li y Merchant 2013).

DRASTIC usa siete variables: D, depth to water (profundidad del agua); $\mathrm{R}$, recharge (recarga neta); $\mathrm{A}$, aquifer media (características del acuífero); $\mathrm{S}$, soil media (suelo); T, topography (topografía); I, impact of the vadose zone media (impacto de la zona vadosa), y $\mathrm{C}$, conductivity (conductividad hidráulica) (Aller et al. 1987). A pesar del amplio uso de DRASTIC en muchas partes del mundo, su eficacia no siempre ha sido exitosa. Se han propuesto diversas modificaciones al índice DRASTIC, las cuales han añadido o excluido diferentes factores según se consideren importantes en la evaluación de la vulnerabilidad; un ejemplo es la inclusión de la cobertura y uso del suelo, pues se considera que las actividades antrópicas pueden modificar la vulnerabilidad natural potencial de las aguas subterráneas a la contaminación (Lima et al. 2011).

La utilización de técnicas de SIG es apropiada para la tarea de evaluación y control del peligro de contaminación del agua subterránea. Estas técnicas facilitan el almacenamiento, actualización, manejo e integración de los datos en forma eficiente, además de una presentación flexible de los resultados a través de mapas (Foster et al. 2002). En este trabajo se aplica un método desarrollado con el apoyo de un SIG, que integra un modelo de vulnerabilidad intrínseca y un inventario de fuentes contaminantes, para la determinación de los índices de peligrosidad y riesgo a la contaminación del agua subterránea en el estado de
Yucatán. La finalidad es proporcionar información acerca de zonas potencialmente amenazadas y establecer una base para desarrollar mejores estrategias, con el objetivo de lograr un manejo sostenible del acuífero y la protección de áreas prioritarias para su recarga.

\section{MATERIALES Y MÉTODOS}

\section{Área de estudio}

El estado de Yucatán ocupa el extremo septentrional de la península que lleva su nombre, y constituye la porción más oriental de México (Fig. 1). Las temperaturas medias anuales del estado se distribuyen de 24 a los $28^{\circ} \mathrm{C}$; hay dos zonas térmicas: la cálida, de 24 a $26^{\circ} \mathrm{C}$, y la muy cálida, con temperaturas mayores a $26^{\circ} \mathrm{C}$. La precipitación media estatal es de $1100 \mathrm{~mm}$ anuales; las lluvias se presentan en verano, de junio a octubre. El subsuelo está constituido por una secuencia de sedimentos calcáreos de origen marino del Terciario reciente, y se compone principalmente de una caliza dura formada por la precipitación de carbonato de calcio. La falta de arcillas y margas sobre la caliza provoca que en periodos de lluvia se infiltre rápidamente el agua, disolviendo la roca y formando un relieve llamado karst o cárstico (García y Graniel 2010).

El agua subterránea en Yucatán es la principal fuente de abastecimiento para las distintas actividades de la población; a su vez, el acuífero es receptor de las aguas de desecho que se generan en el estado. Existen problemas de calidad a causa de la alta permeabilidad del suelo, lo que hace al acuífero muy vulnerable a la contaminación debido a la mala disposición de aguas residuales, fosas sépticas, aguas pluviales y otros factores como lixiviados de tiraderos o basureros municipales. Asimismo, hay otras fuentes de origen natural que modifican la calidad del agua subterránea y la inutilizan para el abastecimiento, como la intrusión salina en la franja costera o la presencia de yesos en algunas áreas del sur del estado (Graniel 2010). Por tal motivo, es muy importante la evaluación de la vulnerabilidad y su integración al análisis del riesgo para la delimitación de zonas muy vulnerables y para la toma de decisiones sobre el uso de suelo.

\section{Análisis de peligros}

Para la identificación y categorización de los peligros de contaminación presentes en la entidad, se siguió el método propuesto por el grupo COST Action 620 (Zwahlen 2003). 


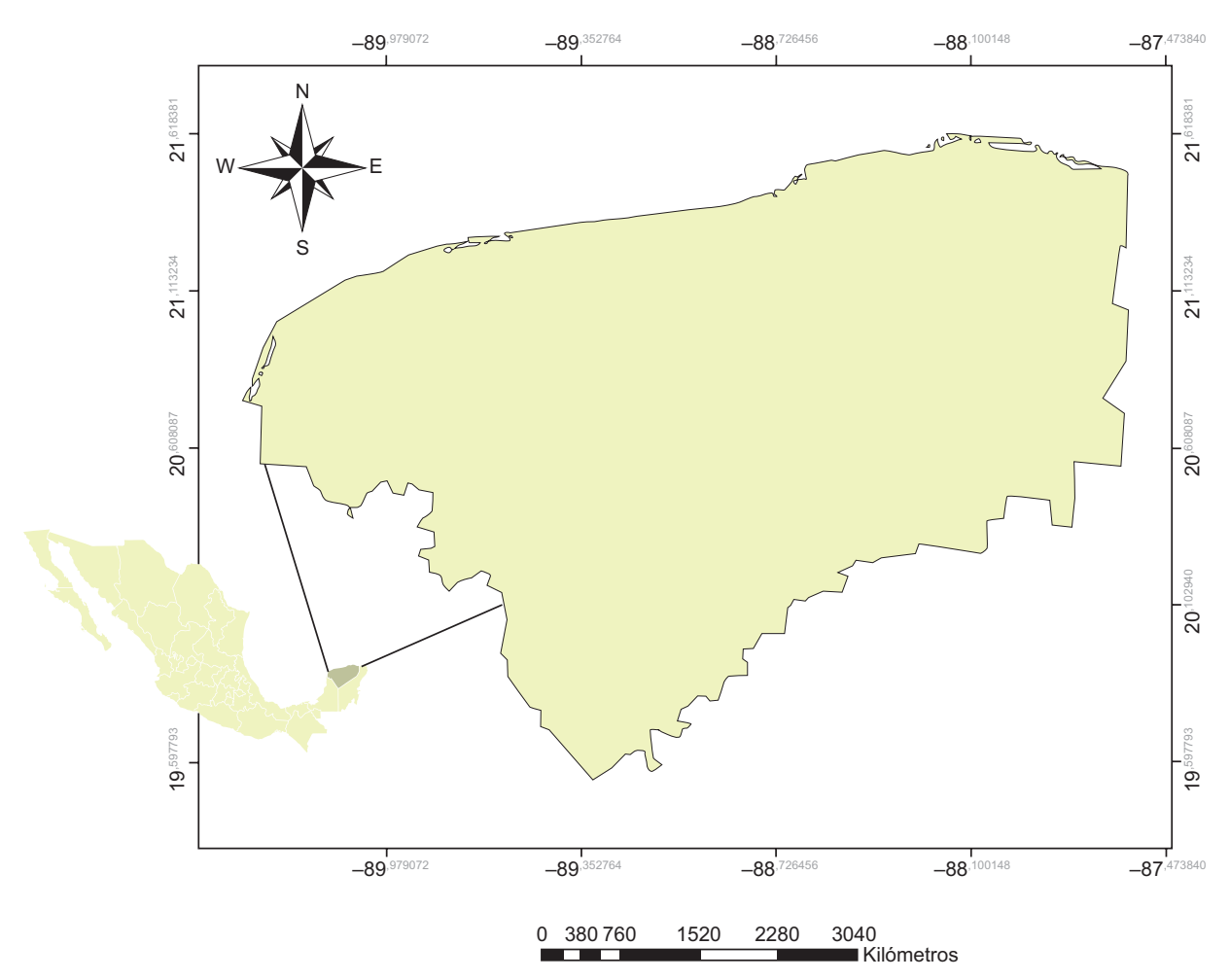

Fig. 1. Localización del área de estudio (estado de Yucatán, México)

El primer paso consiste en realizar el inventario de peligros, que consiste en una diferenciación general sobre una escala regional o local acerca del uso de suelo; en él se distinguen tres categorías: infraestructura, actividades industriales y actividades agrícolas, las cuales se consideran como el nivel I de dicho inventario. Las categorías del nivel II usan un criterio adicional de clasificación: distinguen entre peligros de acuerdo con la posible fuente principal de contaminación (contaminantes líquidos o sólidos), o bien pueden referirse a los tipos de actividades industriales o agrícolas con sus correspondientes espectros de posibles contaminantes. Las categorías consideradas dentro del nivel III representan una subdivisión de las contenidas en el nivel II. Las categorías del nivel III se mencionan con más detalle en el cuadro I (Zwahlen 2003). La información acerca de los peligros se obtuvo de bases de datos y archivos shape provenientes de dependencias de gobiernos municipales, estatales y federales, estudios publicados y mapas de atlas de riesgos.

El segundo paso consiste en la evaluación del grado de peligrosidad para cada tipo de actividad riesgosa. La información colectada para cada tipo de peligro contiene, en la medida de lo posible, la naturaleza de la actividad, localización por coordenadas, caracterización y cuantificación de los residuos sólidos y líquidos.
El tercer paso es la categorización y ponderación de los peligros; los coeficientes de ponderación, que varían de 10 a 100 , se enlistan en el cuadro I. Un peligro del mismo tipo pero con diferentes características se puede comparar usando un factor de rango $\left(Q_{n}\right)$, el cual representa la cantidad o grado de sustancia nociva que puede ser potencialmente liberada o descargada al acuífero, y varía de 0.8 a 1.2 (Mimi y Assi 2009).

Además de considerar el tipo o cantidad de sustancias peligrosas que se asocian a la amenaza, también debe incluirse el nivel técnico, el nivel de mantenimiento, los alrededores y las medidas de seguridad, que son factores importantes cuando se evalúa la probabilidad de que un evento real de contaminación ocurra. Para evaluar esta probabilidad se aplica un coeficiente de reducción $\left(R_{f}\right)$ que varía de 1 a 0 . Si $R_{f}$ es igual a 0 no hay riesgo de contaminación, mientras que un $R_{f}$ igual a 1 indica que no hay información para saber si existe una reducción de la probabilidad de que un evento contaminante ocurra. El índice de peligrosidad $H I$ (hazard index) describe el grado de peligrosidad de cada actividad riesgosa; para su cálculo se usa la siguiente fórmula:

$H I=H^{*} Q_{n}{ }^{*} R_{f}$ 
CUADRO I. PELIGROS Y VALORES DE PONDERACIÓN (ZWAHLEN 2003)

\begin{tabular}{|c|c|c|}
\hline Núm. & Peligros & Ponderación \\
\hline 1 & INFRAESTRUCTURA & \\
\hline \multirow[t]{7}{*}{1.1} & Aguas residuales & \\
\hline & $\begin{array}{l}\text { Urbanización (fugas de pipas y } \\
\text { sistemas de alcantarillado) }\end{array}$ & 35 \\
\hline & $\begin{array}{l}\text { Urbanización sin sistemas de } \\
\text { alcantarillado }\end{array}$ & 70 \\
\hline & $\begin{array}{l}\text { Viviendas unifamiliares sin sistemas } \\
\text { de alcantarillado }\end{array}$ & 45 \\
\hline & Tanques sépticos, pozos, letrinas & 45 \\
\hline & Escorrentías de superficies pavimentadas & 25 \\
\hline & $\begin{array}{l}\text { Descarga de aguas residuales dentro de } \\
\text { corrientes de aguas superficiales }\end{array}$ & 45 \\
\hline \multirow[t]{6}{*}{1.2} & Residuos municipales & \\
\hline & $\begin{array}{l}\text { Tiraderos a cielo abierto, cestos de basu- } \\
\text { ra, papeleras }\end{array}$ & 40 \\
\hline & $\begin{array}{l}\text { Estación de cargas de residuos y depósi- } \\
\text { tos de chatarra }\end{array}$ & 40 \\
\hline & Rellenos sanitarios & 50 \\
\hline & Almacenes y depósitos de escombros & 35 \\
\hline & Lodos de plantas de tratamientos & 35 \\
\hline \multirow[t]{4}{*}{1.3} & Combustibles & \\
\hline & $\begin{array}{l}\text { Tanques de almacenamiento sobre el } \\
\text { suelo }\end{array}$ & 50 \\
\hline & $\begin{array}{l}\text { Tanques de almacenamiento en } \\
\text { el subsuelo }\end{array}$ & 55 \\
\hline & Estaciones de gasolina & 60 \\
\hline \multirow[t]{3}{*}{1.4} & Transporte y tráfico & \\
\hline & Carreteras y caminos & 40 \\
\hline & Tuberías de líquidos peligrosos & 60 \\
\hline \multirow[t]{2}{*}{1.5} & Instalaciones recreativas & \\
\hline & Urbanización a causa del turismo & 30 \\
\hline \multirow[t]{3}{*}{1.6} & Diversos peligros & \\
\hline & Cementerios & 25 \\
\hline & Entierros de animales & 35 \\
\hline
\end{tabular}

CUADRO I. PELIGROS Y VALORES DE PONDERACIÓN (ZWAHLEN 2003) (continuación)

\begin{tabular}{|c|c|c|}
\hline Núm. & Peligros & Ponderación \\
\hline 2 & ACTIVIDADES INDUSTRIALES & \\
\hline \multirow[t]{2}{*}{2.1} & Minas (en operación y abandonadas) & \\
\hline & Minería de sal & 60 \\
\hline \multirow[t]{3}{*}{2.2} & Sitios de excavación & \\
\hline & $\begin{array}{l}\text { Excavaciones y terraplenes para la } \\
\text { infraestructura }\end{array}$ & 10 \\
\hline & Bancos de materiales & 30 \\
\hline \multirow[t]{4}{*}{2.3} & Explotación de aceite y gas & \\
\hline & Pozos de producción & 40 \\
\hline & Pozos de reinyección & 70 \\
\hline & Estaciones de carga & 55 \\
\hline \multirow[t]{5}{*}{2.4} & Plantas industriales & \\
\hline & Obras de hierro y acero & 40 \\
\hline & Industrias químicas & 65 \\
\hline & Curtidurías de cuero & 70 \\
\hline & Industrias de alimentos & 45 \\
\hline \multirow[t]{2}{*}{2.5} & Plantas de energía & \\
\hline & Plantas de energía calórica & 50 \\
\hline \multirow[t]{3}{*}{2.6} & Almacenes industriales & \\
\hline & $\begin{array}{l}\text { Depósitos de materias primas } \\
\text { y productos químicos }\end{array}$ & 60 \\
\hline & Contenedores de sustancias peligrosas & 70 \\
\hline \multirow[t]{4}{*}{2.7} & Desvío y tratamiento de aguas residuales & \\
\hline & Tuberías de aguas residuales & 65 \\
\hline & $\begin{array}{l}\text { Embalses superficiales de aguas } \\
\text { residuales industriales }\end{array}$ & 65 \\
\hline & Descarga de plantas de tratamiento & 40 \\
\hline 3 & GANADERIA Y AGRICULTURA & \\
\hline \multirow[t]{3}{*}{3.1} & Ganadería & \\
\hline & Graneros & 30 \\
\hline & Granjas & 30 \\
\hline \multirow[t]{3}{*}{3.2} & Agricultura & \\
\hline & Ensilados & 25 \\
\hline & Fertilizantes y pesticidas & 40 \\
\hline
\end{tabular}


donde $\mathrm{HI}$ es el índice de peligrosidad, $\mathrm{H}$ es el valor de la ponderación de cada peligrosidad asignado en el cuadro I, $Q_{n}$ es el factor de rango (0.8-1.2) y $R_{f}$ es el factor de reducción (0-1). El rango posible del $H I$ varía de 0 a 120.

El cuarto paso es la producción del mapa de peligros, que es la interpretación gráfica de las fuentes o eventos contaminantes donde se muestra su localización, extensión (tamaño, forma) e información descriptiva.

El quinto paso es la integración de la información sobre los peligros en un SIG para producir el mapa de peligrosidad.

El sexto paso consiste en la evaluación de los datos en cuanto a la calidad de los mismos, el acoplamiento de los modelos en el SIG, los resultados y la sensibilidad de éstos.

El séptimo paso es la producción del mapa de peligrosidad con base en los resultados del índice de peligrosidad aplicado para cada amenaza.

Tanto el mapa de peligros como el mapa del índice de peligrosidad fueron desarrollados con el software ArcGIS 10.1. En el cuadro II se muestran los colores que se utilizan para representar el grado de peligrosidad potencial de las diferentes amenazas en el mapa (Mimi y Assi 2009).

\section{Evaluación de la vulnerabilidad}

Se implementó una modificación del modelo DRASTIC, (DRSTIL) (Ecuación 2) que ha sido aplicado por Albornoz-Eúan y González-Herrera (2017) para evaluar la vulnerabilidad del acuífero de Yucatán, México:

$\pi=D_{R} D_{W}+R_{R} R_{W}+S_{R} S_{W}+T_{R} T_{W}+I_{R} I_{W}+L_{R} L_{W}$

donde $\pi$ es el índice de vulnerabilidad, $D$ es la profundidad del agua, $R$ la recarga, $S$ se refiere al suelo, $T$ corresponde a la topografía, $I$ al impacto de la zona vadosa y $L$ al uso del suelo. Los subíndices $R$ y $W$ son la puntuación y el valor de ponderación otorgado a cada factor, respectivamente.

En este modelo, los factores $A$ (litología del acuífero) y $C$ (conductividad hidráulica) no se toman en cuenta, ya que se relacionan más con la ruta, longitud, transporte, difusión y degradación de los contaminantes considerando todo el sistema acuífero (Aller et al. 1987). Para el área de estudio estos factores no representan grandes variaciones (se considera al acuífero yucateco en su totalidad como un sistema cárstico para el cual González-Herrera et al. [2002] reportan conductividades hidráulicas de $5.5 \times 10^{-3} \mathrm{~m} / \mathrm{s}$ y $1.115 \mathrm{~m} / \mathrm{s}$ obtenidas de un modelo de flujo de aguas
CUADRO II. ÍNDICES DE PELIGROSIDAD Y SUS CLASES (ZWAHLEN 2003)

\begin{tabular}{cccc}
\hline $\begin{array}{c}\text { Índice de } \\
\text { peligrosidad }\end{array}$ & $\begin{array}{c}\text { Clase de } \\
\text { peligrosidad }\end{array}$ & Nivel de peligro & Color \\
\hline $0-24$ & 1 & Ninguno o muy bajo & Azul \\
$>24-48$ & 2 & Bajo & Verde \\
$>48-72$ & 3 & Moderado & Amarillo \\
$>72-96$ & 4 & Alta & Naranja \\
$>96-120$ & 5 & Muy alta & Rojo \\
\hline
\end{tabular}

subterráneas), ya que las puntuaciones para estos factores se basan en los rangos establecidos por el método DRASTIC y los valores que se asignarían serían similares, lo que ocasionaría una redundancia para el cálculo de las diferentes clases de vulnerabilidad.

La aplicación de la modificación DRSTIL resulta ventajosa, ya que excluye estos factores; además, en el presente caso, sólo se requiere evaluar la vulnerabilidad a la contaminación a nivel freático, es decir, la susceptibilidad del sistema a la entrada de contaminantes. Asimismo, se añadió el factor $L$ (uso del suelo) para reflejar los impactos de las diversas actividades, que se desarrollan sobre la superficie del suelo, sobre la calidad del agua subterránea (Li y Merchant 2013); este factor presenta grandes variaciones en el área de estudio, por lo que puede contribuir a una mejor identificación de las áreas con mayor riesgo de ser contaminadas.

El desarrollo de cada uno de los factores del modelo DRSTIL se realizó con ayuda del programa ArcGIS 10.1. En el cuadro III se desglosan las fuentes de datos utilizadas para el desarrollo de cada factor del índice DRSTIL, junto con su respectiva ponderación de acuerdo con el método DRASTIC (Aller et al. 1987), excepto para el factor uso de suelo, cuyas puntuaciones, así como la ponderación asignada, se tomaron con base en diversos estudios en que se incorpora este factor al índice DRASTIC (Rupert 1999, Ceplecha et al. 2004, Kabbour et al. 2004, Lima et al. 2011, Li 2012).

La estimación del factor profundidad del agua se realizó siguiendo el método de configuración del nivel freático propuesta por Snyder (2008) (Fig. 2). La recarga se calculó de acuerdo con el método propuesto por la CONAGUA (2010), según el cual la recarga natural por infiltración corresponde a la diferencia entre el volumen precipitado y el volumen evapotranspirado. Los valores de precipitación y evapotranspiración se obtuvieron de los archivos shape de la CONABIO. Los rangos de precipitación media, así como los datos de evapotranspiración real 
CUADRO III. FUENTE DE DATOS PARA CADA FACTOR DEL ÍNDICE DRSTIL

\begin{tabular}{llc}
\hline Factor & Fuente de datos & Ponderación \\
\hline Profundidad del agua & $\begin{array}{l}\text { Bases de datos de la CONAGUA (con registros hasta 2015) } \\
\text { y modelo digital de elevación (MED) del INEGI }\end{array}$ & 5 \\
\hline Recarga & Archivos de formato shape de la CONABIO (2012) & 4 \\
\hline Suelo & $\begin{array}{l}\text { Archivos de formato shape de la CONABIO (2012) } \\
\text { y guías de interpretación edafológica }\end{array}$ & 2 \\
\hline Topografía & MED del INEGI & 1 \\
\hline $\begin{array}{l}\text { Impacto de la zona } \\
\text { vadosa }\end{array}$ & $\begin{array}{l}\text { Cartas geológicas y archivos de formato shape de } \\
\text { la SEDUMA (2007) }\end{array}$ & 5 \\
\hline Uso de suelo & Archivo de formato shape del INEGI (2014) \\
\hline $\begin{array}{l}\text { CONAGUA: Comisión Nacional del Agua; INEGI: Instituto Nacional de Estadística y Geografía; } \\
\text { CONABIO: Comisión Nacional para el Conocimiento y Uso de la Biodiversidad; SEDUMA: Secretaría } \\
\text { de Desarrollo Urbano y Medio Ambiente del Estado de Yucatán }\end{array}$
\end{tabular}

media anual, se calcularon según el método de Turc con información de 543 estaciones en un periodo de 25 años, según los trabajos realizados por Maderey (1990). Los parámetros para el análisis kriging se determinaron por medio de variogramas desarro- llados con el programa GS+ (Geoestatistics for the Environmental Sciences).

Para la obtención del mapa de vulnerabilidad con el índice DRSTIL, todos los factores fueron estandarizados en forma directa a las puntuaciones

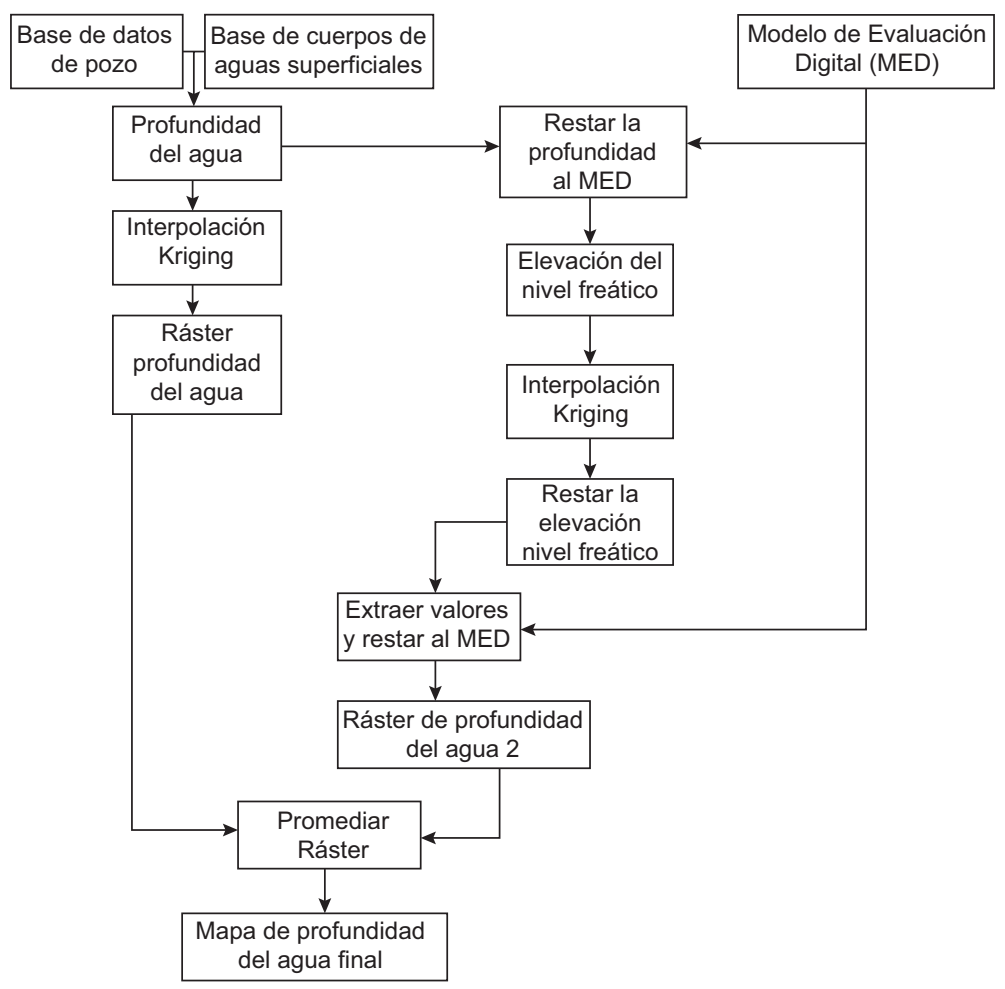

Fig. 2. Método utilizado para la estimación de la profundidad del agua 
del método DRASTIC (Aller et al. 1987) excepto los factores profundidad del agua $(D)$ y recarga $(R)$, los cuales se estandarizaron por medio de ecuaciones derivadas de curvas de ajuste. Los rásteres estandarizados se agregaron por medio de la herramienta Suma ponderada del módulo Análisis espacial de ArcGIS 10.1. El ráster de salida fue reclasificado de acuerdo con los rangos de valores para el índice DRSTIL calculados por medio de la suma de cada una de las puntuaciones ponderadas para cada factor (Cuadro IV).

CUADRO IV. INTERVALOS DE CLASIFICACIÓN DEL ÍNDICE DE VULNERABILIDAD DRSTIL

\begin{tabular}{cc}
\hline Índice DRSTIL & Clasificación \\
\hline $0-65$ & Mínima \\
$>65-120$ & Baja \\
$>120-160$ & Moderada \\
$>160-192$ & Alta \\
$>192$ & Extrema \\
\hline
\end{tabular}

\section{Evaluación y mapa de riesgo}

Para el mapeo del riesgo de contaminación, el enfoque usado en este método se basó en la integración de la carga contaminante en el subsuelo y la vulnerabilidad a la contaminación del acuífero en la locación para la cual se evalúa la intensidad del riesgo. Los mapas realizados con este enfoque muestran el riesgo de contaminación de cada peligro en relación con el recurso de protección (acuíferos). El índice de intensidad de riesgo RII (risk intensity assessment) es la probabilidad de que los contaminantes con una cierta cantidad y concentración alcancen el nivel freático del agua subterránea. El cálculo del índice de intensidad del riesgo considera los efectos de la vulnerabilidad y los peligros usando la siguiente ecuación:

$$
R I I=\frac{I}{H I} * \pi
$$

Donde RII es el índice de intensidad del riesgo, $H I$ es el índice de peligrosidad y $\pi$ es el índice de vulnerabilidad. Los límites de las clases de riesgo son el producto de los límites de las clases de vulnerabilidad y el índice de peligrosidad, como se observa en el cuadro $\mathbf{V}$, lo cual es una ventaja, ya que se trata de una operación muy fácil de implementar que permite la adaptación a los diferentes índices de vulnerabilidad. En el cuadro $\mathbf{V}$ también se puede apreciar la clase a la que pertenece cada riesgo de acuerdo con los resultados del índice de riesgo, así como los colores usados para representar cada nivel de riesgo en el mapa.

\section{RESULTADOS}

\section{Análisis del mapa de peligros}

El mapa de peligros del estado de Yucatán (Fig. 3) permitió identificar zonas de pastizal cultivado e inducido, las cuales ocupan $4972 \mathrm{~km}^{2}(12.71 \%$ del territorio estatal) y se clasificaron como áreas de pastoreo intensivo conforme a los niveles del cuadro III. Asimismo, las zonas de agricultura temporal e intensiva, que ocupan $2926 \mathrm{~km}^{2}$ (7.47\%), se clasificaron como áreas de fertilizantes y plaguicidas, en tanto que las áreas urbanizadas ocupan $380.31 \mathrm{~km}^{2}(0.009 \%)$. La ciudad de Mérida es la que tiene mayor densidad de viviendas, y no cuenta con un sistema de drenaje para aguas residuales, por lo tanto se le clasificó como urbanización sin sistema de alcantarillado. Las demás áreas urbanizadas corresponden al resto de las cabeceras municipales, las cuales se clasificaron como viviendas con tanques sépticos, pozos y letrinas por la menor densidad de viviendas y la menor extensión ocupada. En cuanto a las carreteras, se incluyeron solamente las de mayor aforo vehicular, que cubren $1532.253 \mathrm{~km}$. Las estaciones de gasolina

CUADRO V. ÍNDICE DE RIESGOS Y SUS CLASES

\begin{tabular}{cccccc}
\hline $\begin{array}{c}\text { Índice de } \\
\text { vulnerabilidad }\end{array}$ & $\begin{array}{c}\text { Índice de } \\
\text { peligrosidad }\end{array}$ & $\begin{array}{c}\text { Índice de } \\
\text { riesgo }\end{array}$ & $\begin{array}{c}\text { Clase de } \\
\text { índice }\end{array}$ & $\begin{array}{c}\text { Nivel de } \\
\text { riesgo }\end{array}$ & Color \\
\hline $0-65$ & $0-24$ & $0-1560$ & 1 & Ninguno o muy bajo & Azul \\
$>65-120$ & $>24-48$ & $>1560-5712$ & 2 & Bajo & Verde \\
$>120-160$ & $>48-72$ & $>5712-11448$ & 3 & Moderado & Amarillo \\
$>160-192$ & $>72-96$ & $>11448-18432$ & 4 & Alto & Naranja \\
$>192$ & $>96-120$ & $>18432$ & 5 & Extremo & Rojo \\
\hline
\end{tabular}




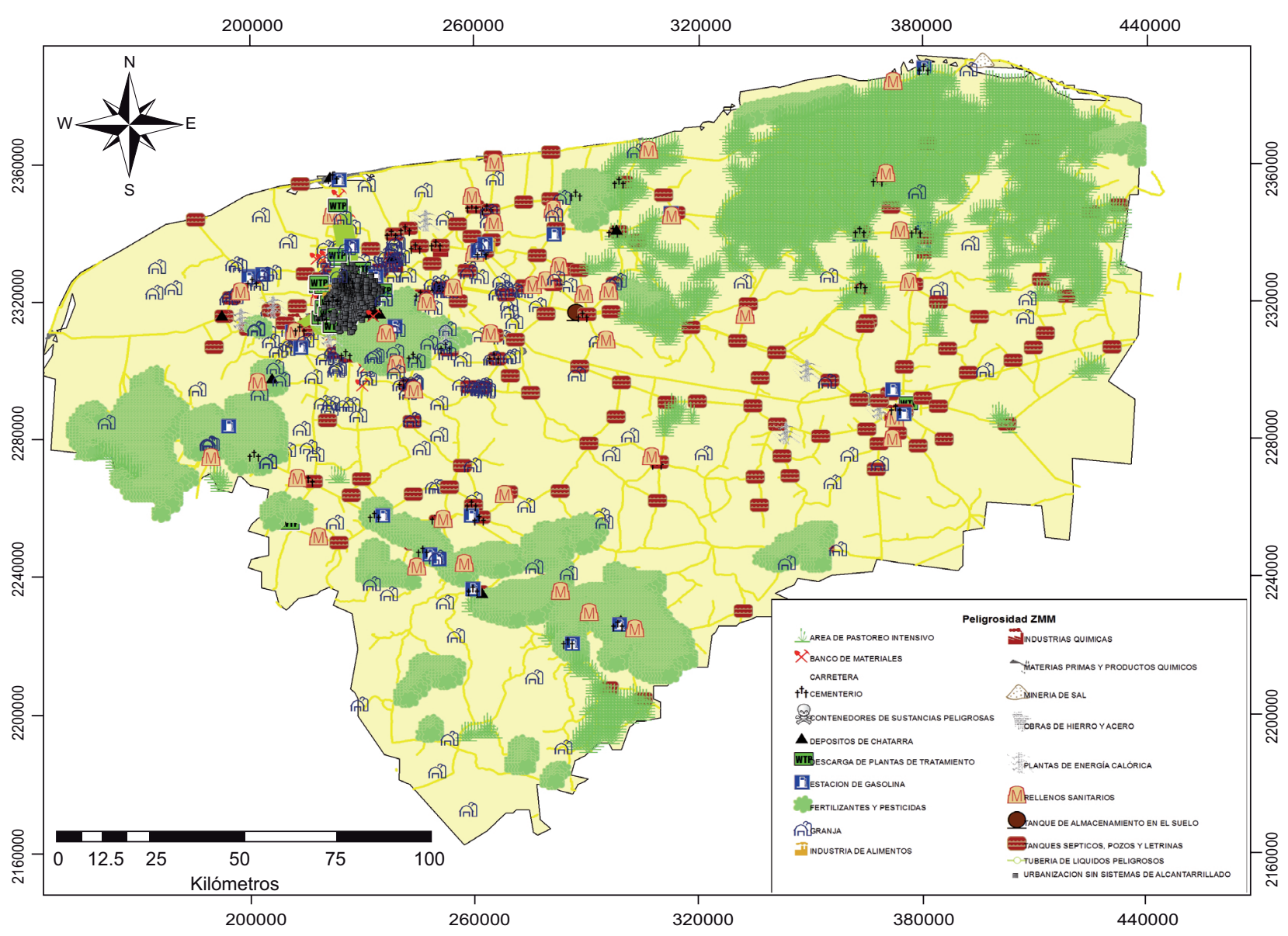

Fig. 3. Mapa de peligros del estado de Yucatán

se encuentran dispersas en todo el territorio estatal, generalmente dentro de las áreas urbanizadas y las carreteras. Las granjas, los bancos de materiales, los cementerios y los rellenos sanitarios se localizan en las inmediaciones de las zonas urbanizadas. Los rellenos sanitarios son de tipo C (de 10 a 50 ton/día), presentes en ocho municipios; de tipo D (menores a 10 ton/día), localizados en 34 municipios, y sólo hay un relleno sanitario de tipo A (mayor a 100 ton/día) en el municipio de Mérida. Estos rellenos se encuentran ubicados espacialmente; en el resto de los municipios existen tiraderos a cielo abierto de cuyas ubicaciones no se tiene un registro exacto.

El factor de ponderación $H$ se determinó conforme a lo propuesto por COST 620 y el factor de rango $Q_{n}$ se evaluó considerando las especificaciones técnicas de cada tipo de amenaza. Debido a que no se cuenta con información acerca del estado de mantenimiento en el que se encuentra la mayoría de los peligros identificados, el factor de reducción $R_{f}$ asignado fue de 1. A ciertos peligros, como las plantas de tratamiento biológico de aguas residuales, se les asignó un $R_{f}$ de 0.6 , de acuerdo con algunas aplicaciones de los mapas de riesgos en determinadas zonas de Alemania realizadas por el grupo COST 620. A los cementerios igualmente se les aplicó un $R_{f}$ de 0.5 , ya que el manejo de las defunciones es parecida a la que se realiza en la Sierra de Líbar, al sur de España (Zwahlen 2003), pues las personas fallecidas no son enterradas sino puestas en ataúdes y colocados en bóvedas. En el cuadro VI se listan todos los peligros encontrados en la entidad y sus respectivos valores de ponderación.

El mapa de peligrosidad (Fig. 4) indica que la clase de peligrosidad alta ocupa una superficie de 104.19 $\mathrm{km}^{2}(0.27 \%)$ y se encuentra ubicada en la ciudad de Mérida, que es la mayor urbe en el estado y no cuenta con sistema de drenaje, por lo que la mayoría de las aguas residuales generadas se descargan en sumideros y fosas sépticas. Asimismo, en dicha área urbana se localizan industrias, almacenes y una gran densidad de estaciones de gasolina, tanques de almacenamiento 
CUADRO VI. AMENAZAS ENCONTRADAS EN EL ESTADO DE YUCATÁN

\begin{tabular}{|c|c|c|c|c|c|c|}
\hline AMENAZAS & $\begin{array}{c}H \\
\text { (factor de } \\
\text { ponderación) }\end{array}$ & $\begin{array}{c}Q_{n} \\
\text { (factor de } \\
\text { rango) }\end{array}$ & $\begin{array}{c}R_{f} \\
\text { (factor de } \\
\text { reducción) }\end{array}$ & $\begin{array}{c}H I \\
\text { (índice de } \\
\text { peligrosidad) }\end{array}$ & $\begin{array}{c}\pi \\
\text { (índice de } \\
\text { vulnerabilidad) }\end{array}$ & $\begin{array}{c}\text { RII } \\
\text { (índice } \\
\text { de riesgo) }\end{array}$ \\
\hline \multicolumn{7}{|l|}{ Agricultura } \\
\hline Áreas de pastoreo intensivo & 25 & 1 & 1 & 25 & $66-194$ & $1650-4850$ \\
\hline Fertilizantes y plaguicidas & 40 & 1 & 1 & 40 & $63-196$ & $2520-7840$ \\
\hline \multicolumn{7}{|l|}{ Aguas residuales } \\
\hline $\begin{array}{l}\text { Tanques sépticos, pozos y } \\
\text { letrinas }\end{array}$ & 45 & $0.8-1.2$ & 1 & $36-45-54$ & $81-180$ & $1.5-5$ \\
\hline $\begin{array}{l}\text { Urbanización sin sistema } \\
\text { de alcantarillado }\end{array}$ & 70 & 1.2 & 1 & 84 & $149-187$ & $2.8-15624$ \\
\hline \multicolumn{7}{|l|}{ Almacenes industriales } \\
\hline $\begin{array}{l}\text { Contenedores de sustancias } \\
\text { peligrosas }\end{array}$ & 70 & 1 & 1 & 70 & $153-172$ & $2.1-2.4$ \\
\hline $\begin{array}{l}\text { Materias primas y productos } \\
\text { químicos }\end{array}$ & 60 & 1 & 1 & 60 & $155-170$ & $2.5-2.8$ \\
\hline \multicolumn{7}{|l|}{ Combustibles } \\
\hline Estaciones de gasolina & 60 & $1-1.2$ & 1 & $60-72$ & $78-183$ & $2-13726$ \\
\hline $\begin{array}{l}\text { Tanques de almacenamiento en } \\
\text { el suelo }\end{array}$ & 50 & 1 & 1 & 50 & $50-182$ & $3-3.1$ \\
\hline \multicolumn{7}{|c|}{ Desvío y tratamiento de aguas residuales } \\
\hline $\begin{array}{l}\text { Descarga de plantas de } \\
\text { tratamiento de aguas residuales }\end{array}$ & 40 & $0.8-1.2$ & 0.6 & $19.2-24-28.8$ & $73-186$ & $3.8-5011.2$ \\
\hline \multicolumn{7}{|l|}{ Diversos peligros } \\
\hline Cementerios & 25 & $0.8-1$ & 0.5 & $10-12.5$ & $100-175$ & $12.64-1925$ \\
\hline \multicolumn{7}{|l|}{ Ganadería } \\
\hline Granjas & 30 & $0.8-1.2$ & 1 & $24-30-36$ & $70-195$ & $1.9-5310$ \\
\hline \multicolumn{7}{|l|}{ Minería } \\
\hline Minería de sal & 60 & 1.2 & 1 & 72 & 127 & 1.7 \\
\hline \multicolumn{7}{|l|}{ Plantas de energía } \\
\hline Plantas de energía calórica & 50 & 1.2 & 1 & 60 & $92-182$ & $2.2-10920$ \\
\hline \multicolumn{7}{|l|}{ Plantas industriales } \\
\hline Industrias de alimentos & 45 & 1 & 1 & 45 & $126-186$ & $2.8-7965$ \\
\hline Industrias químicas & 65 & $1-1.2$ & 1 & $65-78$ & $128-172$ & $1.6-12792$ \\
\hline Obras de hierro y acero & 40 & 1 & 1 & 40 & 182 & 4.55 \\
\hline \multicolumn{7}{|l|}{ Residuos municipales } \\
\hline Depósitos de chatarra & 40 & $1-1.2$ & 1 & $40-48$ & $99-186$ & $3960-8928$ \\
\hline Rellenos sanitarios & 50 & $0.8-1.2$ & 1 & $40-50-60$ & $70-181$ & $2.8-9050$ \\
\hline \multicolumn{7}{|l|}{ Sitios de excavación } \\
\hline Bancos de materiales & 30 & 1 & 1 & 30 & $125-182$ & $4.1-4590$ \\
\hline \multicolumn{7}{|l|}{ Transporte } \\
\hline Carretera & 40 & $0.8-1.2$ & 1 & $32-40-48$ & $58-196$ & $1.45-6.12$ \\
\hline $\begin{array}{l}\text { Tuberías de líquidos peligrosos } \\
\text { (oleoductos) }\end{array}$ & 60 & 1.2 & 1 & 72 & $138-184$ & $2.4-13248$ \\
\hline
\end{tabular}




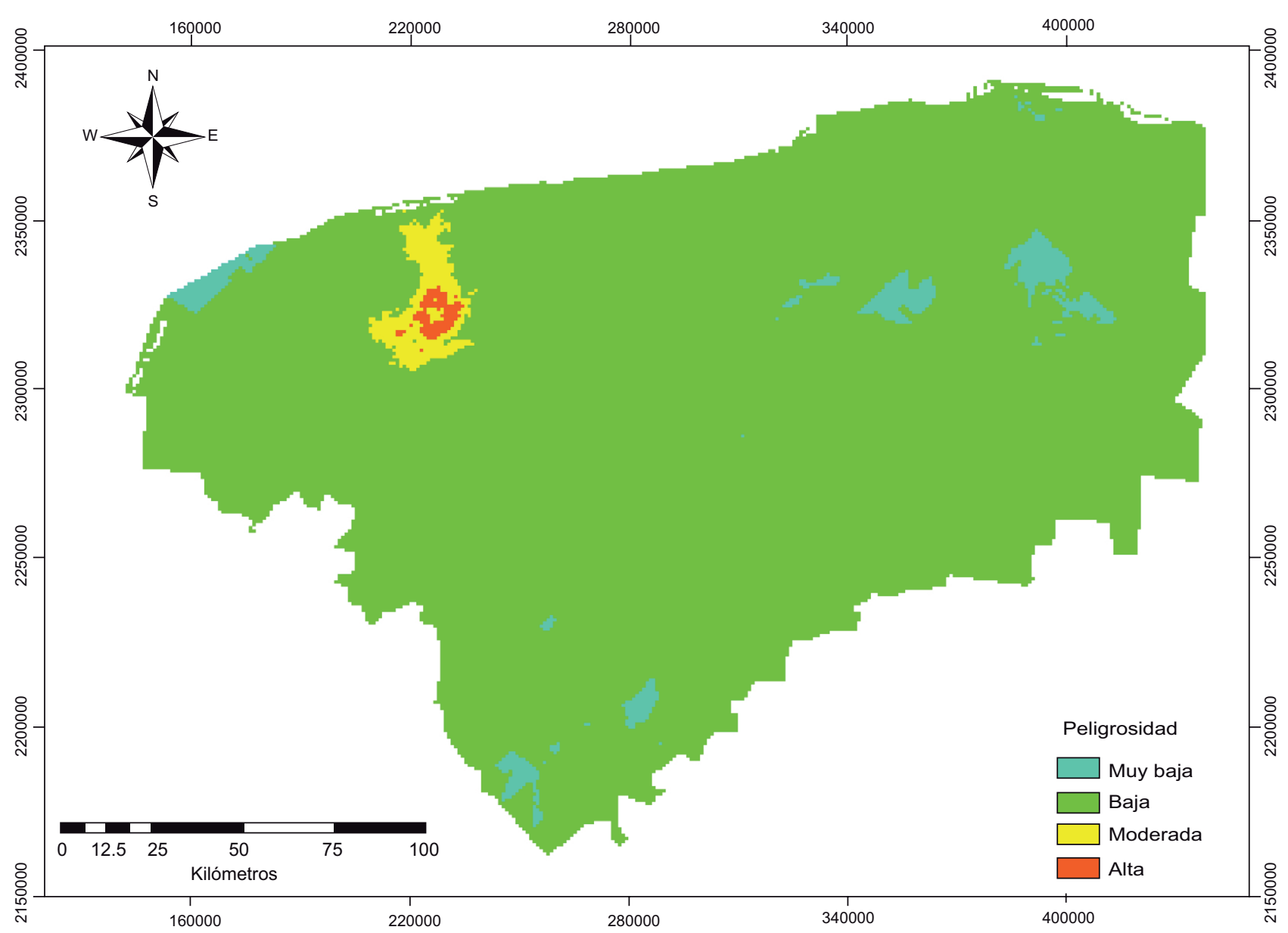

Fig. 4. Clases de peligrosidad en el estado de Yucatán

de combustibles, depósitos de chatarra y plantas de tratamiento de aguas residuales procedentes de hoteles, oficinas y nuevos fraccionamientos. La clase de peligrosidad moderada se encuentra alrededor de la ciudad de Mérida, en la cual también se asientan desarrollos inmobiliarios con plantas de tratamiento para sus aguas residuales y algunas industrias; sin embargo, éstas se encuentran más dispersas.

\section{Análisis del mapa de vulnerabilidad}

Los valores del índice de vulnerabilidad DRSTIL para el estado de Yucatán fluctuaron entre 58 y 197. Se obtuvieron cinco clases de vulnerabilidad, como se puede apreciar en la figura 5. mínima, baja, moderada, alta y extrema. Algunas áreas del sur del estado, donde se encuentra ubicada la Sierrita de Ticul, fueron clasificadas con vulnerabilidad mínima, cubriendo aproximadamente un $1.23 \%$ de la superficie total. La vulnerabilidad baja se manifiesta en la mayor parte de la región sur, en algunas zonas del oriente y la región poniente de la zona costera, con un $37.95 \%$ de la extensión total. La clasificación de vulnerabilidad moderada fue la que tuvo mayor cobertura en el estado; se distribuyó principalmente en grandes áreas del oriente, noreste, centro, occidente y poniente, con un $42.51 \%$ del total. Las áreas clasificadas como de alta vulnerabilidad ocupan 18 $\%$ del área total, y se encuentran principalmente en la parte norte de las regiones noreste, litoral centro, occidente y poniente. Por último, la clase de vulnerabilidad extrema fue la de menor extensión, con un $0.31 \%$ del total, y está localizada en la parte sur de la región oriente del estado.

Los resultados obtenidos difieren de los reportados en otros trabajos en los que se implementó el índice DRASTIC o una modificación del mismo. Utilizando el índice DRASTIC, Pérez-Ceballos et al. (2008) encontraron que el $0.2 \%$ de la superficie total de la entidad se clasificó en la categoría de vulnerabilidad moderada, el $61 \%$ como vulnerabilidad alta, el 


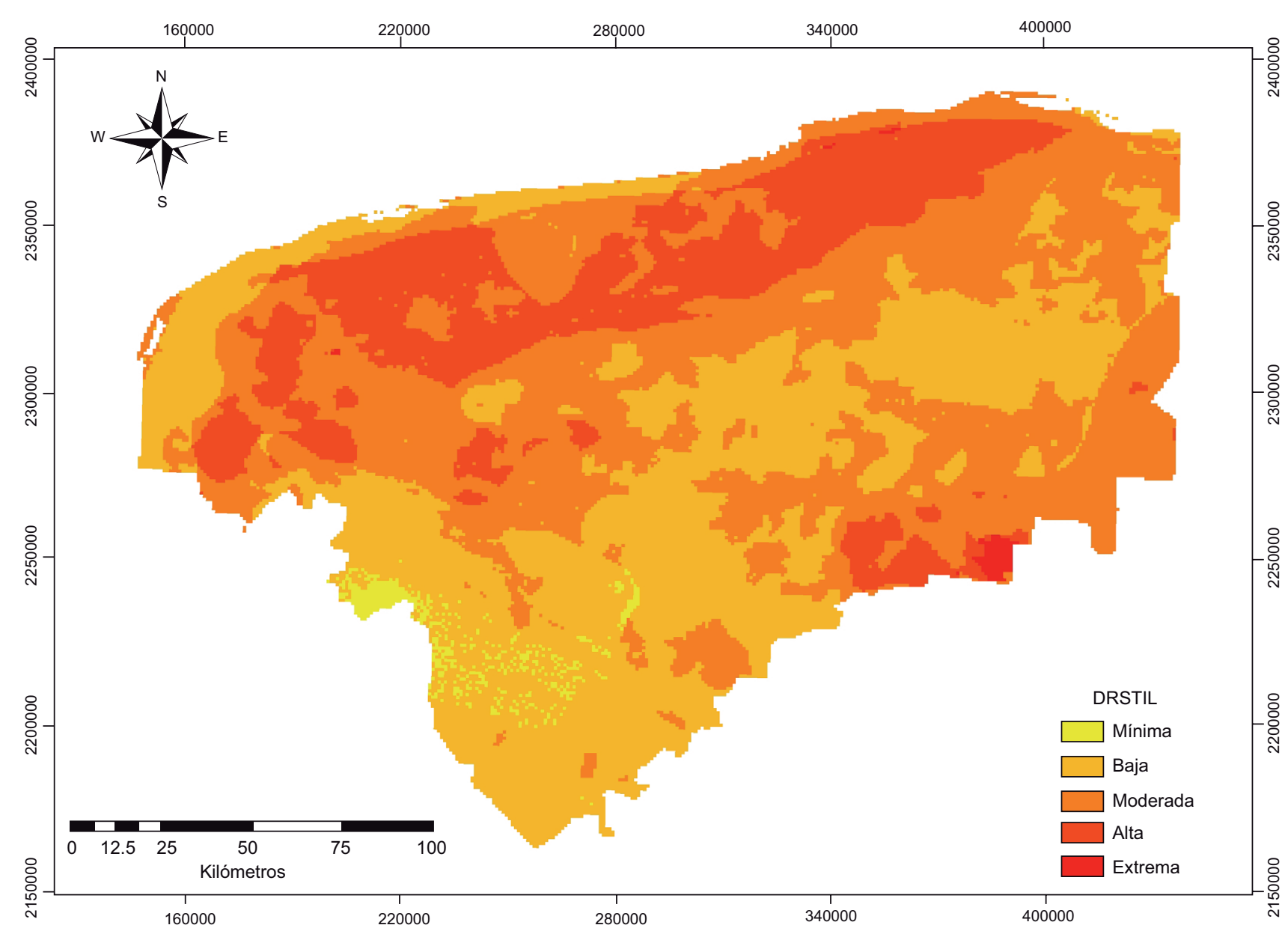

Fig. 5. Mapa de vulnerabilidad del estado de Yucatán con base en el índice DRSTIL

$33.8 \%$ como vulnerabilidad muy alta y el $5 \%$ como vulnerabilidad extrema. Utilizando una modificación del DRASTIC (DSTI), Gijón et al. (2009) clasificaron el $15.48 \%$ de la superficie en la categoría de vulnerabilidad moderada, el $35.98 \%$ en la categoría de vulnerabilidad alta y el $48.54 \%$ en la categoría de vulnerabilidad extrema.

\section{Análisis del mapa de riesgo}

El mapa de riesgo se elaboró con el índice de intensidad de riesgo, el cual varió de 1 a 13 404. Los niveles de riesgo para el área de estudio se clasificaron como muy bajo, bajo, moderado y alto. En la figura 6 se puede observar que las áreas de muy bajo riesgo ocupan una superficie de $14674.44 \mathrm{~km}^{2}(37.43 \%$ del área total de la entidad), la clase de bajo riesgo ocupa $22655.47 \mathrm{~km}^{2}(57.7 \%)$, la clase de riesgo moderado tiene una superficie de $1797.77 \mathrm{~km}^{2}(4.59 \%)$ y la clase de alto riesgo tiene una extensión de $78.8 \mathrm{~km}^{2}(0.20 \%)$. En las zonas clasificadas como de muy bajo riesgo se encuentran principalmente granjas dispersas, algunas urbanizaciones pequeñas y carreteras con bajo aforo vehicular; la mayoría de las zonas coinciden con áreas de vulnerabilidad moderada, aunque en el litoral centro, la vulnerabilidad es alta. En las zonas clasificadas como de bajo riesgo dominan las áreas agrícolas (ya sean de temporal, de riego o pastizales), que se encuentran localizadas principalmente en áreas de vulnerabilidad baja en las regiones del sur y oriente del estado, aunque en la región del noreste la vulnerabilidad es moderada y alta.

Las zonas de riesgo moderado se localizan en algunas áreas de la región oriente, poniente y en la zona metropolitana de Mérida (ZMM). En la región oriente y poniente, este nivel de riesgo se debe principalmente a las actividades agrícolas desarrolladas en un área de vulnerabilidad extrema y alta, respectivamente. Las zonas de alto riesgo se encuentran ubicadas en la ZMM, la cual se encuentra dominada por la urbanización, estaciones de gasolina, numerosas plantas 


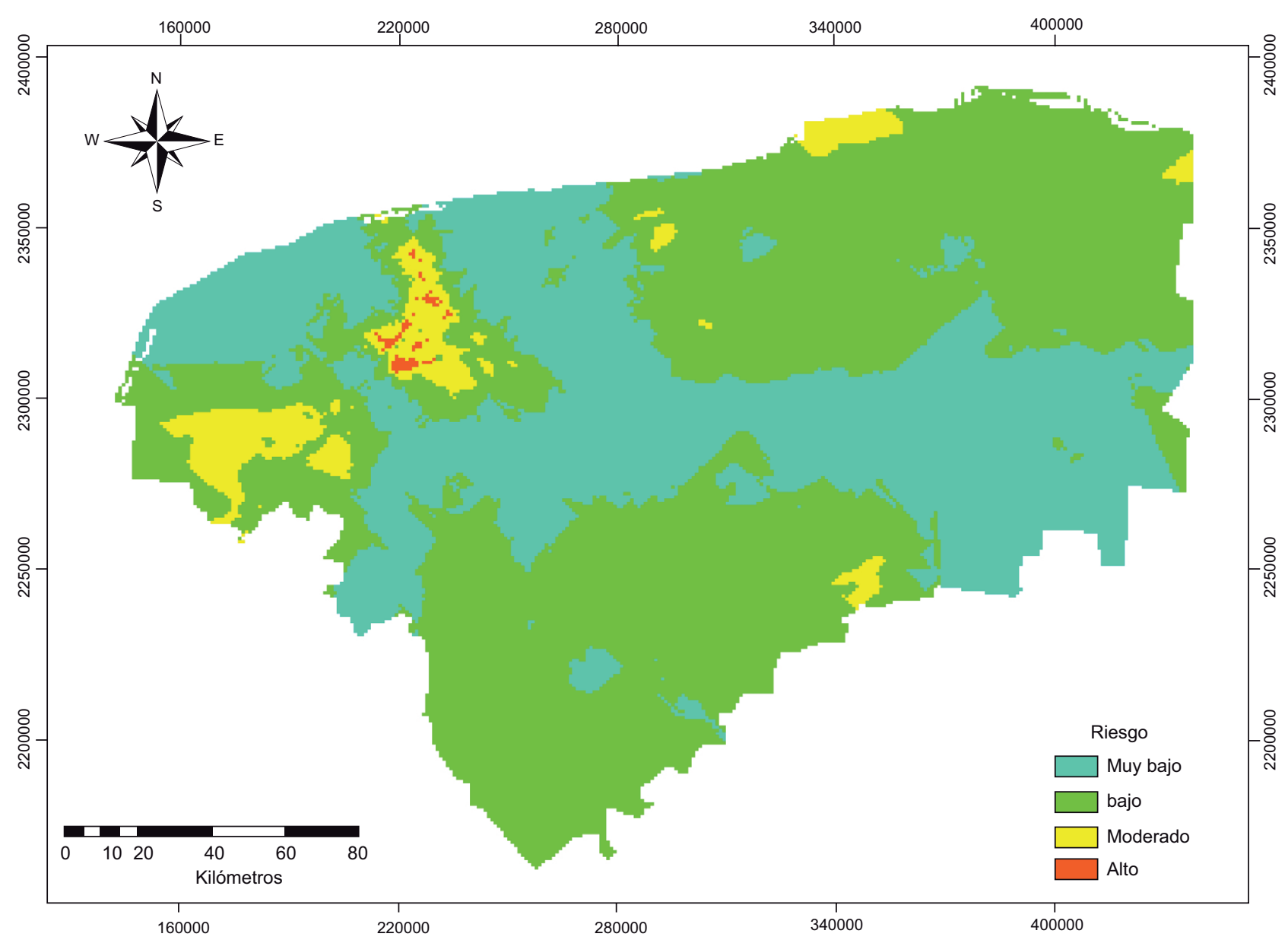

Fig. 6. Clases de riesgos en el estado de Yucatán

industriales, plantas de energía, bancos de materiales y carreteras con gran aforo vehicular, peligros que están asentados en su totalidad en áreas clasificadas como de vulnerabilidad alta. Es necesario destacar que las clasificaciones en el mapa de riesgo pueden cambiar si existen cambios en las características de los peligros, ya sea de incremento o reducción de las posibles sustancias tóxicas liberadas, o bien de la extensión de los mismos. De igual manera, es necesario indicar que el riesgo también se encuentra en función de las clasificaciones de vulnerabilidad, la cual puede cambiar debido a las variaciones de cada uno de los factores que la componen, como la profundidad del nivel freático y la recarga, mismas que pueden sufrir variaciones en el tiempo. Los resultados del mapa de intensidad del riesgo indican áreas problemáticas donde se deben concentrar los esfuerzos para reducir los peligros existentes, así como para prevenir o minimizar la probabilidad tanto de que ocurra un evento contaminante como sus consecuencias por medio de la implementación de acciones correctivas.

\section{DISCUSIÓN}

Tanto la evaluación del riesgo a la contaminación como la creación de mapas temáticos relevantes son de mucha utilidad para la gestión de las aguas subterráneas. Los mapas temáticos representan diversos aspectos que deben considerarse cuando se toman decisiones en la administración de aguas subterráneas. El mapa de peligros puede usarse para distinguir las clases de peligrosidad de las diferentes fuentes contaminantes (Wang et al. 2012); en el caso del estado de Yucatán, la alta peligrosidad se centra en la ciudad de Mérida y su zona metropolitana. Un acercamiento a esta última (Fig. 7) muestra que en ella se asienta la mayor diversidad de fuentes contaminantes, lo cual 


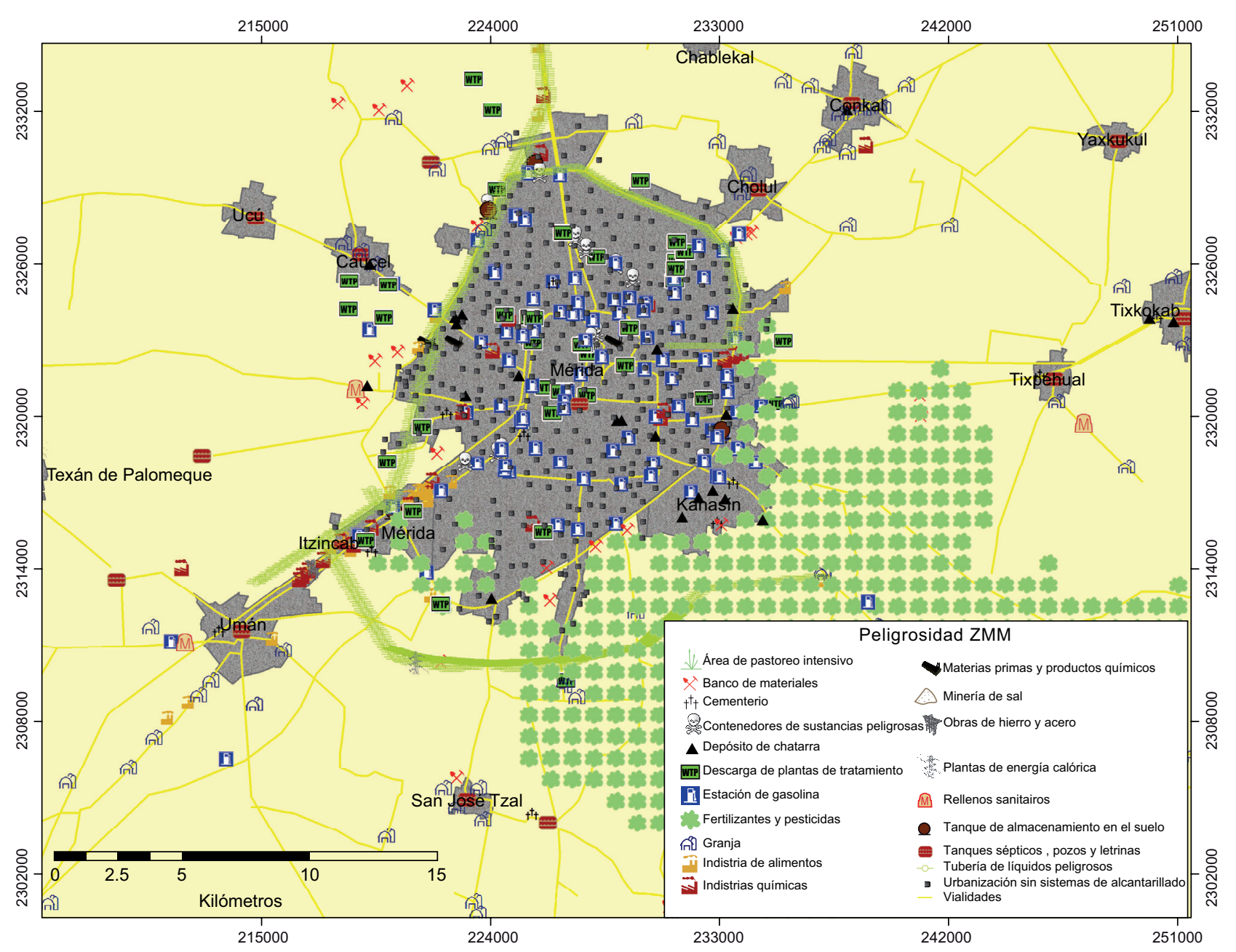

Fig. 7. Mapa de peligros de la zona metropolitana de Mérida

significa que el grado de urbanización es un factor importante que influye en la generación y diversificación de las fuentes de contaminación potencial.

Cuando se analiza un mapa de peligrosidad es necesario tener en cuenta que dentro de las urbanizaciones podrían existir otros peligros que no pueden localizarse espacialmente, como la acumulación de basura en calles, las descargas clandestinas de aceites $\mathrm{u}$ otros líquidos peligrosos, y pequeños criaderos de animales de traspatio. La ausencia de una referencia geográfica, aunada a la falta de conocimiento sobre las características de la amenaza, provoca que estos peligros no puedan incluirse en el cálculo del índice de peligrosidad. Por lo tanto, las urbanizaciones, en general, pueden parecer menos peligrosas de lo que realmente son.

El mapa de vulnerabilidad muestra que las zonas clasificadas como de vulnerabilidad mínima coinciden con áreas donde se registran las mayores profundidades al agua subterránea, los porcentajes más altos de pendiente, parches de suelos con textura arcillosa y calizas estratificadas de varios centenares de metros de espesor. La clasificación de vulnerabilidad baja coincide con las zonas monticulares y de ondulaciones donde se presentan acuíferos a mediana profundidad, además de que los suelos existentes corresponden a texturas de margas arcillosas. Igualmente, en el litoral costero se localiza un estrato confinante que corresponde al caliche costero prácticamente impermeable (Perry et al. 1989); además, en esta zona se presentan suelos arenosos con porciones de arcilla y limo, así como áreas con vegetación natural. En todas estas zonas los valores de recarga no superan los $125 \mathrm{~mm}$.

La clasificación de vulnerabilidad moderada se presenta en zonas donde las profundidades al agua subterránea fluctúa entre 0 y $30 \mathrm{~m}$, los suelos son de textura margo arcillosa-limosa a arenosa, las calizas 
son de alta permeabilidad, las pendientes cercanas al $0 \%$ y la recarga varía de 133 a $280 \mathrm{~mm}$. El uso de suelo es muy variado; hay zonas con agricultura temporal, de pastizal cultivado, pastizal inducido y algunos parches de vegetación natural.

Las áreas clasificadas como de alta vulnerabilidad se localizan en zonas planas donde se presentan acuíferos a poca profundidad (de 0 a 14 metros), suelos de textura margo limosa-arenosa, pendientes de $0 \%$, calizas de alta permeabilidad (formación Carrillo Puerto), valores de recarga superiores a 300 $\mathrm{mm}$ y grandes extensiones de agricultura temporal y de pastizal cultivado e inducido. Estas últimas características no hacen sino elevar los valores de vulnerabilidad; por lo tanto, se clasifican como altos. La clasificación de vulnerabilidad extrema se presenta en zonas donde la profundidad al agua subterránea varía de 0 a 8 metros, la recarga es superior a los 300 $\mathrm{mm}$, los suelos son de textura margo limosa-arenosa, la inclinación de pendientes tiene valores muy cercanos a 0 y calizas de alta permeabilidad, todo lo cual no hace sino extremar el índice de vulnerabilidad en esta área, la cual se encuentra impactada por la agricultura de temporal.

En el mapa de riesgo se puede observar que, en áreas de riesgo moderado, las actividades agrícolas constituyen el principal uso del suelo, lo cual demuestra que si bien la agricultura no es la principal actividad económica del estado, su carácter como fuente de contaminación difusa debido a la aplicación de fertilizantes orgánicos e inorgánicos, así como de plaguicidas, contribuye de manera importante a la contaminación del agua subterránea, sobre todo si se desarrolla en áreas de alta vulnerabilidad. A diferencia de las acciones que pueden emplearse para controlar la contaminación de una fuente puntual, las medidas para mitigar o prevenir los riesgos de fuentes agrícolas difusas son más complejas (Zaporozec 2002). En el estado de Yucatán, la falta de información sobre la cantidad y tipos de plaguicidas y fertilizantes utilizados en los cultivos, dificulta la cuantificación del factor $Q_{n}$ y el coeficiente $R_{f}$. Por otro lado, la falta de capacitación del usuario provoca la mala eliminación final de los desechos y obstaculiza la implementación de alguna medida de mitigación o prevención del arrastre y lixiviación de los contaminantes hacia el agua subterránea.

Cuando se analizan los niveles de peligrosidad y riesgo con el método COST Action 620, se debe tener en cuenta la variación de los coeficientes de ponderación para los peligros, los cuales fluctúan de 0 a 100 . Los coeficientes de valor más alto corresponden a los sitios de eliminación de desechos nucleares, residuos peligrosos y minería de elementos radiactivos como el uranio. Por lo tanto, una zona donde se presente este tipo de peligros con coeficientes de ponderación alto tendrá altos niveles de peligrosidad.

Otro aspecto que se debe considerar es que el enfoque del método COST Action 620 es general y no toma en cuenta las características específicas de algún sitio en particular, además de que el mapa sólo representa la situación actual del área, ya que tanto los tipos de peligro como el grado de peligrosidad pueden cambiar en el futuro por el desarrollo de la industria e infraestructura en el estado, así como el incremento de la población.

Todos los índices de peligrosidad se basan en la experiencia científica. La clasificación del grado de nocividad de cada peligro se sustenta en interpretaciones y suposiciones debido a la falta de información sobre las características y la extensión de las amenazas (von Heike 2006). Contar con información precisa sobre la localización de los peligros y la probabilidad de ocurrencia de un evento contaminante es importante para la protección y la gestión del agua subterránea; por consiguiente, el mapa de peligrosidad en combinación con el mapa de vulnerabilidad es de utilidad para llevar a cabo la evaluación de la intensidad del riesgo con el objetivo de prevenir y reducir los peligros existentes.

A diferencia de otros métodos para clasificar y evaluar peligros, como los métodos Pollutant Origin, Surcharge Hydraulically (POSH) de Foster e Hirata (1988) y Environmental Risk Inventory System (ERIS) de Wilson (1991), los cuales requieren que se conozca cantidad y características específicas de las sustancias contaminantes, el método del grupo COST Action 620 cubre todos los peligros relevantes para el agua subterránea y permite, a través de una subdivisión lógica, el mapeo y la evaluación de manera fácil y económica. Otra de las ventajas que fundamentan el uso del método COST Action 620 en el área de estudio, son los conceptos de ponderación y el factor de rango, los cuales proporciona un esquema para la comparación cuantitativa de los peligros; igualmente, la inclusión de un factor de reducción permite evaluar la probabilidad de ocurrencia de un evento contaminante.

\section{CONCLUSIONES}

En este trabajo se presentó un método que integra un modelo de vulnerabilidad intrínseca y un inventario de fuentes contaminantes, desarrollado con un SIG, para la determinación de los índices de 
peligrosidad y riesgo de contaminación del agua subterránea en el estado de Yucatán. La identificación, clasificación y valoración de los peligros se realizó con el método del grupo COST ACTION 620. Se identificaron 17 peligros que corresponden a áreas urbanizadas, de pastoreo intensivo y de fertilizantes y plaguicidas, así como a carreteras, estaciones de gasolina, granjas, plantas industriales y depósitos de chatarra, entre otros. La ZMM presentó la peligrosidad más alta, ya que en ella se asienta una gran cantidad de fuentes contaminantes.

Para elaborar el mapa de riesgo se utilizó el índice de intensidad de riesgo. Los niveles de riesgo para el área de estudio se clasificaron como muy bajo, bajo, moderado y alto. La región que corresponde a la ZMM se encuentra dominada por la urbanización, las estaciones de gasolina, numerosas plantas industriales, plantas de energía, bancos de materiales y carreteras con gran aforo vehicular, los cuales se asientan sobre áreas clasificadas como de vulnerabilidad alta, por lo tanto son zonas que representan un alto riesgo. Los resultados del mapa de riesgo indican la existencia de áreas problemáticas donde se deben concentrar los esfuerzos para reducir los peligros existentes, así como prevenir o minimizar, por medio de la implementación de acciones correctivas, la probabilidad de que un evento contaminante ocurra $\mathrm{y}$ tenga consecuencias graves.

\section{AGRADECIMIENTOS}

Los autores agradecen a la Universidad Autónoma de Yucatán y al Consejo Nacional de Ciencia y Tecnología por el apoyo otorgado a través del Programa al Desarrollo y Consolidación de los Cuerpos Académicos (PADECCA) para el desarrollo del presente trabajo, y por la beca otorgada a Beth sua Albornoz Euán durante sus estudios de posgrado. Asimismo, a los revisores anónimos cuyos valiosos comentarios contribuyeron a mejorar este manuscrito.

\section{REFERENCIAS}

Albornoz-Euán B. y González-Herrera R. (2017). Vulnerabilidad a la contaminación del acuífero yucateco bajo escenarios de cambio climático. Ecosist. Recur. Agropec. 4 (11), 275-286. DOI: 10.19136/era.a4n11.1037

Aller L., Bennett T., Lehr J.H., Petty R.J. y Hackett G. (1987). DRASTIC: A standardized system for evaluating groundwater pollution potential using hydrogeologic setting. U.S. Environmental Protection
Agengy. Report 600/02-85/018. Washington, D.C., EUA, $177 \mathrm{pp}$.

Ceplecha Z.L., Waskom R.M., Bauder T.A., Sharkoff J.L. y Khosla R. (2004). Vulnerability assessments of Colorado groundwater to nitrate contamination. Water Air Soil Poll. 159, 373-394.

DOI: 10.1023/B:WATE.0000049188.73506.c9

CONAGUA (2010). Diagnóstico hídrico de la Región Hidrológica Administrativa XII Península de Yucatán. Comisión Nacional del Agua, Secretaría de Medio Ambiente y Recursos Naturales, 592 pp.

Foster S. e Hirata R. (1988). Groundwater pollution risk assessment: A methodology using available data. Manual técnico. WHO-PAHO/HPE-CEPIS, Lima, Perú, $81 \mathrm{pp}$.

Foster S., Hirata R., Gomes D., D’Elia M. y Paris M. (2002). Protección de la calidad del agua subterránea. Guía para empresas de agua, autoridades municipales y agencias ambientales. 2da. ed. Groundwater Management Advisory Team GW-MATE, Banco Mundial, Washington D.C., EUA, pp. 115 pp.

García G.G. y Graniel E.C. (2010). Geología. En: Biodiversidad y desarrollo humano en Yucatán (Durán R. y Méndez M., Eds). CICY, PPD-FMAM, CONABIO, SEDUMA, México, pp. 4-6.

Gijón N., Pacheco J, Euán J. y Pérez R. (2009). Análisis espacial de la vulnerabilidad y riesgo del agua subterránea a la contaminación en el estado de Yucatán, México. En: Libro de resúmenes del seminario análisis de la vulnerabilidad y riesgo de contaminación de las aguas subterráneas en la península de Yucatán (Bautista F., Quintana P., Aguilar Y., Pacheco J. y Cabañas D., Eds.). Centro de Investigaciones en Geografía Ambiental, Universidad Nacional Autónoma de México, México.

González-Herrera R., Sánchez-Pinto I. y Gamboa-Vargas J. (2002). Groundwater-flow modeling in the Yucatan karstic aquifer, Mexico. Hydrogeol. J. 10 (5), 539-552. DOI 10.1007/s10040-002-0216-6

Graniel E.C. (2010). Hidrología. En: Biodiversidad y Desarrollo Humano en Yucatán (Durán R. y Méndez M., Eds). CICY, PPD-FMAM, CONABIO, SEDUMA, México, pp. 12-13.

Hudak, P. (2005). Principles of Hydrogeology. 3ra. Ed. CRC Press LLC, Florida, USA. 248 p.

Kabbour B.B., Zouhri L., Mania J. y Colbeaux J.P. (2004). Assessing groundwater contamination risk using the DASTI/IDRISI GIS method: Coastal system of western Mamora, Morocco. Bull. Eng. Geol. Environ. 65 (4), 463-470. DOI: 10.1007/s10064-004-0265-2

Li R. (2012). Groundwater pollution risk assessment under scenarios of climate and land use change in the northern Great Plains. ETD collection for University of Nebraska, AAI3503015. Lincoln, Nebraska, EUA, 156 p. 
Li R. y Merchant J.W. (2013). Modeling vulnerability of groundwater to pollution under future scenarios of climate change and biofuels-related land use change: A case study in North Dakota, USA. Sci. Total Environ. 447, 32-45.

DOI: $10.1016 / j . s c i t o t e n v .2013 .01 .011$

Lima M.L., Zelaya K. y Massone H. (2011). Groundwater vulnerability assessment combining the DRASTIC and DYNA-CLUE model in the Argentine Pampas. Environ. Manage. 47 (5), 828-839.

DOI: $10.1007 / \mathrm{s} 00267-011-9652-1$

Maderey L.E. (1990). Evapotranspiración real. En: Hidrogeografía IV.6.6. Atlas Nacional de México, vol. II. Instituto de Geografía, UNAM, México [en línea]. http://www.conabio.gob.mx/informacion/gis/.

Mimi Z.A. y Assi A. (2009). Intrinsic vulnerability, hazard and risk mapping for karst aquifers: A case study. J. Hydrol. 364 (3), 298-310.

DOI: $10.1016 /$ j.jhydrol.2008.11.008

NRC. (1993). Groundwater vulnerability assessment. Predicting relative contamination potential under conditions of uncertainty. National Research Council, Washington D.C, EUA, 224 pp.

DOI: $10.17226 / 2050$

Nobre R.C.M., Rotunno Filh O.C., Mansur W.J., Nobre M.M.M. y Cosenza, C.A.N. (2007). Groundwater vulnerability and risk mapping using GIS, modeling and a fuzzy logic tool. J. Contam. Hydrol. 94 (7), 277-292. DOI: 10.1016/j.jconhyd.2007.07.008

NSW (1998). Aquifer risk assessment report. New South Wales Department of Land and Water Conservation, Sydney, Australia, $20 \mathrm{pp}$.

Pérez-Ceballos R., Pacheco-Ávila J. y Euán-Ávila J. (2008). Evaluación a escala regional de la vulnerabilidad del agua subterránea a la contaminación en Yucatán, México. Revista Ambiente Ecológico (14), 1-18 [en línea]. http://www.ambiente-ecologico. com/ ediciones/informesEspeciales/014_InformesEspeciales_EvaluacionEscalaRegionalVulnerabilidadAguaSubterraneaContaminacionYucatanMexico.php3 .

Perry E.E., Swift J., Gamboa J., Reeve A., Sanborn R., Marín L.E. y Villasuso M. (1989). Geological and environmental aspects of surface cementation, north coast, Yucatan, Mexico. Geology 17 (9), 818-821. DOI: 10.1130/0091-7613(1989)017<0818:GAEAO $\mathrm{S}>2.3 . \mathrm{CO} ; 2$
Rupert M.G. (1999). Improvements to the DRASTIC groundwater vulnerability mapping method. U.S. Geological Survey, Idaho, EUA, 6 pp.

Snyder D.T. (2008). Estimated depth to ground water and configuration of the water table in the Portland, Oregon área. U.S. Scientific investigations report 2008-5059. U.S. Geological Survey, Reston, Virginia, EUA, 40 pp.

Troldborg M. (2010). Risk assessment models and uncertainty estimation of groundwater contamination from point sources. Ph.D. thesis. Technical University of Denmark, Lyngby, Dinamarca, 89 pp.

Twarakavi N. y Kaluarachchi J. (2006). Sustainability of groundwater quality considering land use changes and public health risks. J. Environ. Manage. 81 (4), 405419. DOI: $10.1016 /$ j.jenvman.2005.11.008

Von Heike W. (2006). The use of remote sensing imagery for groundwater risk intensity mapping in the Wadi Shueib, Jordan. Zur Erlangung des akademischen Grades eines Doktors der Naturwissenschaften an der Fakultät für Bauingenieur- Geo- und Umweltwissenschaften der Universität Karlsruhe genehmigte Dissertation.

Wang J., He J. y Chen H. (2012). Assessment of groundwater contamination risk using hazard quantification, a modified DRASTIC model and groundwater value, Beijing Plain, China. Sci. Total Environ. 432 (15), 216226. DOI: $10.1016 /$ j.scitotenv.2012.06.005

Wilson A. (1991). Environmental risk: Identification and management. C.R. Press, 440 pp.

Zaporozec A. (2002). Groundwater contamination inventory. A methodological guide. UNESCO, París, Francia, 160 pp. (Series on Groundwater No. 2.)

Zwahlen F. (2003). COST ACTION 620. Vulnerability and risk mapping for the protection of carbonate (karst) aquifers. Final report. European Cooperation in Science and Technology, European Commission, Bruselas, Bélgica, 297 pp. 